\title{
Ewing Sarcoma
}

National Cancer Institute

\section{Source}

National Cancer Institute. Ewing Sarcoma. NCI Thesaurus. Code C4817.

A small round cell tumor that lacks morphologic, immunohistochemical, and electron microscopic evidence of neuroectodermal differentiation. It represents one of the two ends of the spectrum called Ewing sarcoma/peripheral neuroectodermal tumor. It affects mostly males under age 20 , and it can occur in soft tissue or bone. Pain and the presence of a mass are the most common clinical symptoms. 